\title{
Effects of a sex-ratio distorting endosymbiont on mtDNA variation in a global insect pest Ana M Delgado ${ }^{1}$ and James M Cook*1,2
} Address: ${ }^{1}$ Division of Biology, Imperial College London, Silwood Park Campus, Ascot, UK and ${ }^{2}$ School of Biological Sciences, University of
Reading, Whiteknights, Reading, UK

Email: Ana M Delgado - ana.delgado01@imperial.ac.uk; James M Cook* - j.cook@imperial.ac.uk

* Corresponding author

Published: 3 March 2009

BMC Evolutionary Biology 2009, 9:49 doi:10.1/86/147I-2148-9-49

This article is available from: http://www.biomedcentral.com/I47I-2/48/9/49

(C) 2009 Delgado and Cook; licensee BioMed Central Ltd.

This is an Open Access article distributed under the terms of the Creative Commons Attribution License (http://creativecommons.org/licenses/by/2.0), which permits unrestricted use, distribution, and reproduction in any medium, provided the original work is properly cited.
Received: 12 August 2008

Accepted: 3 March 2009

\begin{abstract}
Background: Patterns of mtDNA variation within a species reflect long-term population structure, but may also be influenced by maternally inherited endosymbionts, such as Wolbachia. These bacteria often alter host reproductive biology and can drive particular mtDNA haplotypes through populations. We investigated the impacts of Wolbachia infection and geography on mtDNA variation in the diamondback moth, a major global pest whose geographic distribution reflects both natural processes and transport via human agricultural activities.
\end{abstract}

Results: The mtDNA phylogeny of 95 individuals sampled from 10 countries on four continents revealed two major clades. One contained only Wolbachia-infected individuals from Malaysia and Kenya, while the other contained only uninfected individuals, from all countries including Malaysia and Kenya. Within the uninfected group was a further clade containing all individuals from Australasia and displaying very limited sequence variation. In contrast, a biparental nuclear gene phylogeny did not have infected and uninfected clades, supporting the notion that maternallyinherited Wolbachia are responsible for the mtDNA pattern. Only about $5 \%$ (I5/306) of our global sample of individuals was infected with the plutWBI isolate and even within infected local populations, many insects were uninfected. Comparisons of infected and uninfected isofemale lines revealed that plutWBI is associated with sex ratio distortion. Uninfected lines have a I:I sex ratio, while infected ones show a 2:I female bias.

Conclusion: The main correlate of mtDNA variation in P. xylostella is presence or absence of the plutWBI infection. This is associated with substantial sex ratio distortion and the underlying mechanisms deserve further study. In contrast, geographic origin is a poor predictor of moth mtDNA sequences, reflecting human activity in moving the insects around the globe. The exception is a clade of Australasian individuals, which may reflect a bottleneck during their recent introduction to this region.

\section{Background}

Patterns of within-species variation in animal mtDNA are influenced by various factors, including mutation, selection, demography and geography, and analysis of haplo- type diversity patterns can provide information on population structure and gene flow. In addition, mtDNA sequences are often used to investigate the evolutionary history of a species, by combining geographic and phylo- 
genetic information in phylogeographic studies [1]. However, theory predicts that mtDNA variation and evolution may also be influenced substantially by endosymbionts that are maternally co-inherited with the mitochondria [2]. In insects, maternally-inherited intracellular Wolbachia bacteria are of particular concern, because they have been detected in hundreds of species [3] and are estimated to infect about $2 / 3$ of all insect species [4]. Wolbachia cause various modifications of host reproductive biology, including cytoplasmic incompatibility (CI), parthenogenesis, feminisation and male-killing $[5,6]$. In addition, a single infection can be responsible for causing more than one phenotype, e.g. CI and male-killing [7]. Wolbachia enhance their own spread through the host population by means of these phenotypes and, in so doing, drive the associated host mitochondrial haplotypes to high frequencies, causing dramatic changes in host mtDNA patterns [8]. Consequently, analysis of variation in insect mtDNA should take account of possible endosymbiont effects whilst attempting to uncover and explain patterns in terms of host ecology $[2,8]$.

Here, we explore the role of Wolbachia bacteria in explaining patterns of mtDNA variation in the diamondback moth (Plutella xylostella), a global pest of Brassica plants (cabbages and relatives). A previous study [9] reported the occurrence of two different Wolbachia isolates in two different $P$. xylostella individuals during a general screening of diverse insects for Wolbachia infections; however, there have been no wider studies of the occurrence of these isolates in P. xylostella or of their effects on host phenotypes and genetic variation. Consequently, we screened individuals from four continents for Wolbachia infections and then investigated the correlation between infection with the dominant isolate (plutWB1) and mtDNA variation. We also sequenced a biparentally inherited nuclear gene marker, whose phylogeny should reflect demography and geography, but not any Wolbachia-associated driving of cytoplasmic factors. In addition, we conducted experiments to test whether plutWB1 produces a phenotypic effect on the host that is likely to drive the infection, and its associated mtDNA haplotype, through local populations.

The diamondback moth is amongst the most globally distributed of all Lepidoptera [10]. It is generally thought to be endemic to the Mediterranean region [11], although an African origin has also been suggested [12]. Its current global distribution is due to two processes - natural migrations and man-made introductions. Direct observations using vertical-looking radar have confirmed that long distance migration from continental Europe is the source of populations in the UK in late spring [13]. Meanwhile, transport to many disparate locations across the globe is likely to be the result of accidental introductions during vegetable shipments [14]. Consequently, we predict an unusually weak correlation between geographic and genetic (mtDNA) divergence in $P$. xylostella because recent human activity has moved moths between continents.

In summary, we screened $P$. xylostella samples from four continents in order to: (1) determine the global diversity and prevalence of Wolbachia infections, (2) test whether Wolbachia infections were associated with particular mtDNA haplotypes, (3) test whether plutWB1 produces a drive phenotype, 4) test for a breakdown of the usual strong link between geographic continent of origin and mtDNA sequence variation, and 5) compare mtDNA results with those from a nuclear marker that should not have its phylogeny shaped by cytoplasmic drive mechanisms.

\section{Results}

\section{Global screening for Wolbachia in P. xylostella}

In order to explore the prevalence and diversity of Wolbachia, we screened 306 individuals from 10 countries on four continents (Table 1) and isolated three Wolbachia isolates, termed plutWA1, plutWA2 and plutWB1 (Figure 1). The first two isolates were extremely rare and found in only two individuals each, while plutWB1 was found in about 5\% (15/306) of the global sample of individuals (Table 1). We detected Wolbachia only in individuals from Malaysia, Kenya and Germany. However, even in these countries, most individuals were uninfected (Table 1).

\section{Moth mtDNA phylogeny}

We obtained mtDNA sequences for 92 P. xylostella individuals and three members of the closely related outgroup species Plutella porrectalla (Table 1). The mtDNA fragment analysed consisted of $637 \mathrm{bp}$ of the $\mathrm{CO} 1$ gene. There were 72 variable nucleotide sites, of which 55 were informative for maximum parsimony (MP) analyses. This variation

Table I: Global screening of Wolbachia infections in Plutella xylostella.

\begin{tabular}{cccc}
\hline Country & N screened & N infect. (\%) & Infections \\
\hline Malaysia & 122 & $10(8.2)$ & plutWBI, plutWAI \\
Australia & 73 & 0 & \\
Kenya & 41 & $5(12.2)$ & plutWBI \\
UK & 24 & 0 & \\
S Africa & 6 & 0 & \\
Taiwan & 10 & 0 & \\
Hawaii & 10 & 0 & plutWA2 \\
Sweden & 5 & 0 & \\
Germany & 5 & $2(40)$ & \\
New Zealand & 10 & 0 & \\
\hline Total & 306 & 15 & \\
\hline
\end{tabular}




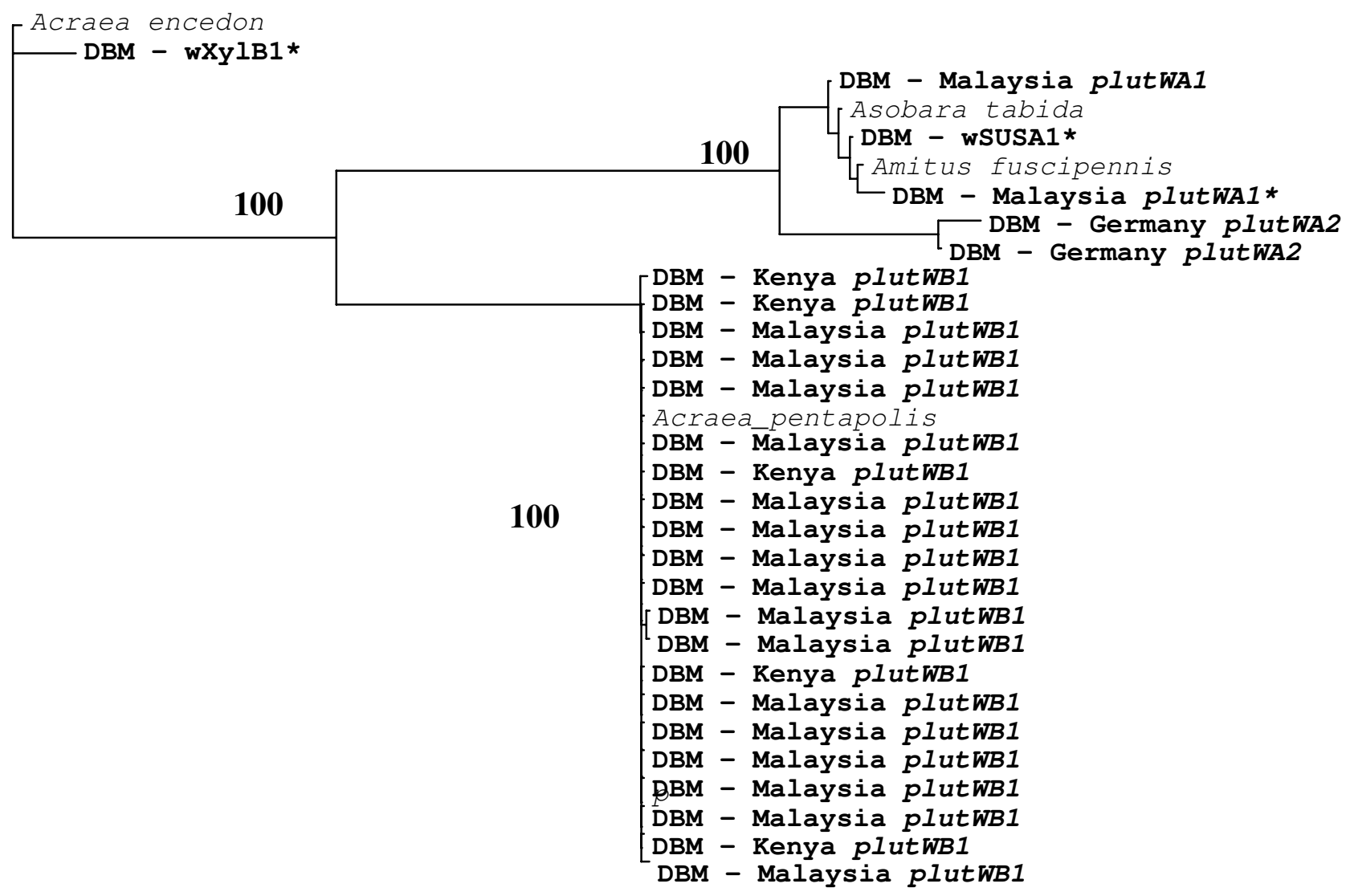

Figure I

Phylogeny of Wolbachia sequences from P. xylostella hosts. Neighbour-joining tree of Wolbachia wsp sequences from Plutella xylostella (country of origin) and other insects. Bootstrap values $>65 \%$ are shown. $*$ denotes Wolbachia isolates found infecting P. xylostella in a previous study [9]. Sequences have been deposited in Genbank with accession numbers EU833334833358.

yielded 22 discrete haplotypes (Figure 2), of which 11 where unique to one individual.

$\mathrm{MP}$ and neighbour-joining (NJ) phylogenies were very similar and revealed a deep split $(2.5 \%$ sequence divergence) between all individuals infected with plutWB1 and all those that were not (Figure 2). In contrast, the largest pairwise distance between any two uninfected individuals was only $1.75 \%$. We sequenced 21 individuals from Kenya and Malaysia that were infected with plutWB1. They all had very similar COI sequences, with maximum pairwise divergence of only $0.314 \%$, forming haplotypes $1-3$ and a well-supported monophyletic clade (Figure 2 ). This supports a single origin of the infection and we further tested for an association between mtDNA haplotypes and plutWB1 by randomising the infection status of individuals across the tips of the phylogeny and calculating the minimum number of changes in infection status implied (see [15], for a similar application). All of the 100 randomisations implied more than the one change observed in the actual data set, providing significant support $(\mathrm{P}<$ 0.01 ) for the association between infection status and haplotype that is apparent by eye. In contrast to the clear pattern found with this isolate, the two A clade Wolbachia isolates were each found in only two individuals, precluding further useful analysis.

In general, individuals do not cluster on the phylogeny according to their geographic origin. However, all individuals from Australia (Haplotypes 7, 8 and 13) and New Zealand (Haplotype 7), along with one from Malaysia (Haplotype 7) form a well-supported monophyletic clade (Figure 2). Australasia was the best sampled region, with individuals from 14 sites spanning most of Australia, as well as two sites in New Zealand.

\section{MtDNA haplotype analysis}

Haplotype and nucleotide diversity estimates for different geographic regions are presented in Table 2. Nucleotide diversity $(\pi)$ for each region is generally low but ranges 


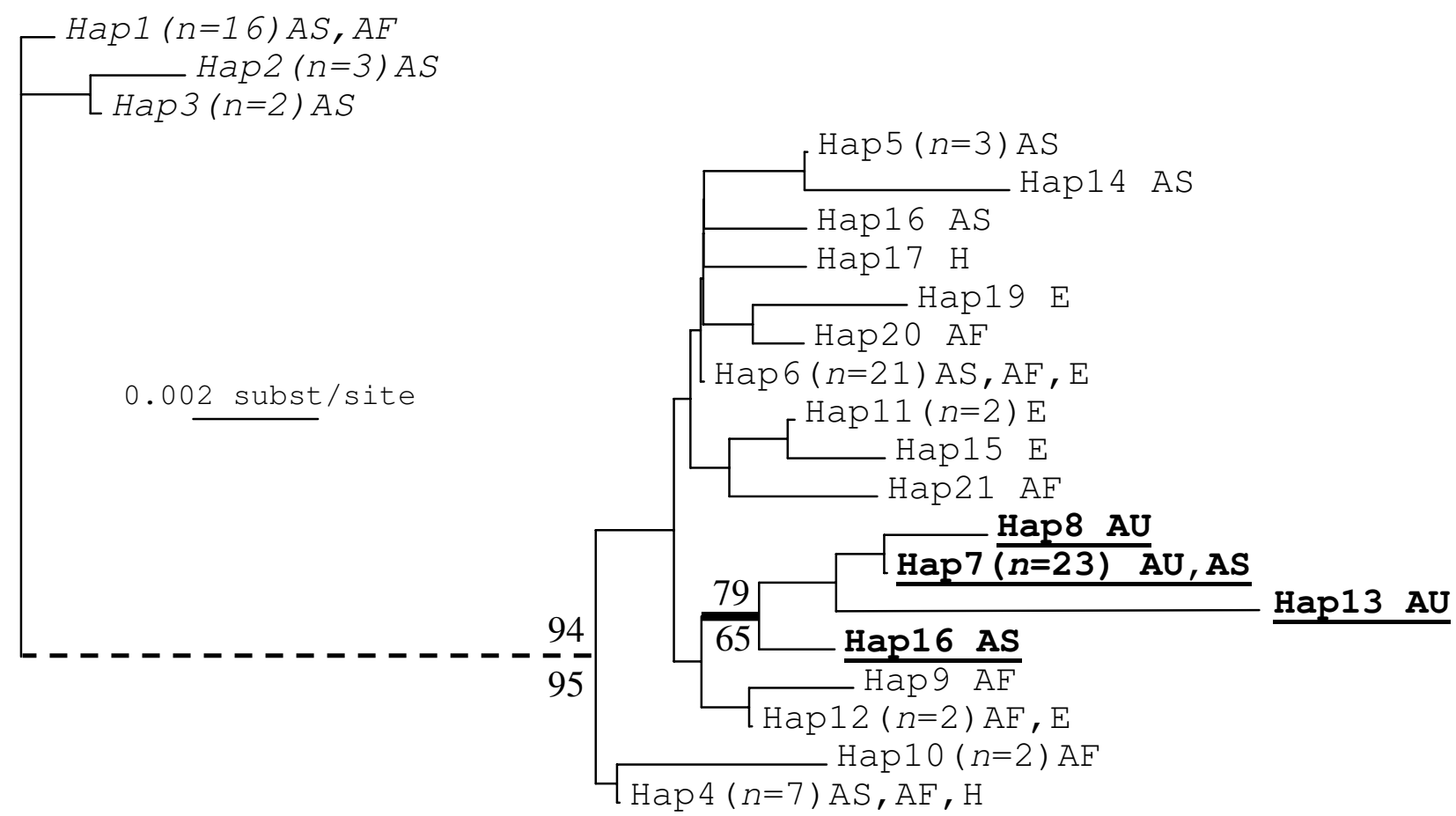

Figure 2

Phylogeny of $\boldsymbol{P}$. xylostella moths using mtDNA data. Neighbour-joining tree of $P$. xylostella mtDNA (COI) haplotypes. Numbers in brackets show individuals with a given haplotype. Geographic occurrences are shown as Asia (AS), Africa (AF), Australasia $(A U)$, Europe $(E)$ and Hawaii $(H)$. The broken line shows that the long branch between Clade $A$ (italics; all $2 \mathrm{I}$ individuals infected with PlutWBI) and Clade B (normal text; all 7I not infected) is not to scale. A further Clade $C$ (all 26 individuals from Australasia) is shown in bold. Sequences have been deposited in Genbank with accession numbers EU833237-833257.

Table 2: Haplotype and nucleotide diversity of COI sequences from different geographical regions and infection categories.

\begin{tabular}{ccccccc}
\hline & N & NHap & DHap & S & $\pi$ & SD $(\pi)$ \\
\hline Region (sites) & & & & & & \\
Australasia (16) & 22 & 5 & 0.338 & 8 & 0.0013 & 0.0007 \\
Asia (6) & 31 & 10 & 0.839 & 17 & 0.0093 & 0.0006 \\
Africa (6) & 14 & 9 & 0.934 & 15 & 0.0091 & 0.0010 \\
Europe (5) & 17 & 5 & 0.507 & 5 & 0.0012 & 0.0004 \\
Hawaii (I) & 4 & 2 & 0.500 & 2 & 0.0015 & 0.0008 \\
& & & & & & \\
\hline Group & & & & & & \\
All Infected & 21 & 3 & 0.4 & 2 & 0.0009 & 0.0003 \\
All Uninfected & 71 & 18 & 0.8 & 22 & 0.00324 & 0.00031 \\
Malaysia -Infected & 18 & 3 & 0.508 & 2 & 0.0015 & 0.00032 \\
Malaysia Uninfected & 12 & 7 & 0.864 & 9 & 0.00317 & 0.00071 \\
Kenya Infected & 5 & 2 & 0.6 & 1 & 0.00093 & 0.00028 \\
Kenya Uninfected & 8 & 6 & 0.893 & 6 & 0.00237 & 0.00059
\end{tabular}

Key: $\mathrm{N}=$ No. individuals; $\mathrm{NHap}=$ No. Haplotypes; $\mathrm{DHap}=$ Haplotype diversity; $S=$ No. of variable sites; $\pi=$ nucleotide diversity.

from 0.0013 to 0.091 . The highest nucleotide diversity was found in Asia, followed by Africa; importantly, these are the two regions that harboured the plutWB1 infection and its associated divergent haplotypes. Australasia showed the lowest haplotype diversity and nucleotide diversity, despite field sampling from 14 sites across Australia and two in New Zealand. This is reflected further by comparisons with data from Hawaii (Table 2). Hawaii, like Australasia, is isolated by sea from other continents and was probably only colonised relatively recently by $P$. xylostella due to human travel. Only four individuals from Hawaii were sampled, but these had higher haplotype diversity than the Australasian individuals and values similar to those in Europe. All infected groups have lower haplotype number, and haplotype and nucleotide diversity, than their respective uninfected groups (Table 2).

\section{Moth nuclear DNA}

We also analysed approximately 200 bp of the L27a nuclear ribosomal protein gene for the same 92 P. xylostella individuals. We obtained 74 different sequences (Genbank accession numbers EU833259-833333), 65 of which were unique to one individual. However, there was low sequence divergence and the vast majority of pairs of individuals differed by only 1-4 nucleotide substitutions. Further, many substitutions were unique and therefore not phylogenetically informative. Nevertheless, two clades can be defined by the presence/absence of a $9 \mathrm{bp}$ indel event. Clade A contained 74 individuals from all five 
continents, while clade $\mathrm{B}$ contained 20 individuals from Europe, Asia and Africa (Figure 3). In contrast to the case for mtDNA, there were not separate infected and uninfected clades. A mixture of infected and uninfected individuals occurred in each clade. All individuals from Australasia were in clade A, indicating that Australasian populations have reduced nuclear diversity compared to most other countries (present in both clades), as suggested by Endersby [14], based on microsatellite markers.

\section{Sequence variation in the Wolbachia wsp gene}

The plutWB1 isolate found in our study shows high sequence divergence (17.3-17.5\%) from the B-clade Wolbachia isolate $(w X y l-B 1)$ previously identified infecting $P$. xylostella [9] (Figure 1). However, it has the same wsp sequence (0-0.2\% sequence divergence) as an isolate found infecting the African butterfly, Acraea pentapolis [16].

\section{Phenotypic effects of Wolbachia}

We found a female biased sex ratio of approximately 0.62 (proportion females) in field P. xylostella populations in the Cameron Highlands, Malaysia (Table 3). The sex ratio was only significantly different from $1: 1$ for the site with the largest sample (or using pooled data from all sites). However, the sex ratio was actually remarkably consistent (0.60-0.63) between the four sites surveyed in this region (Table 3).

We successfully established two infected and three uninfected isofemale lines from field-caught Malaysian $P$. xylostella individuals and maintained them in the laboratory for nine generations (see methods). The two infected lines had female biased mean sex ratios $(0.68,0.66$, Table 4) that were similar but slightly more biased than those observed in the field populations (see above) and these did not change significantly over nine generations of laboratory culture $(F=3.634$, d.f. $=2, P>0.05)$. In contrast, the uninfected lines had unbiased sex ratios $(0.5,0.54$, 0.52 ) (Table 4). The plutWB1 infection appears to be a sex ratio distorter that increases the proportion of female hosts, as infected lines have significantly higher proportions of females than uninfected lines $(t=8.82$, d.f. $=3, P$ $<0.01)$.

The proportion of infected individuals in infected lines decreased over the nine generations $(F=63.66$, d.f. $=2, P$ $<0.05$ ), suggesting that vertical transmission of Wolbachia is not perfect. This was also found when both sexes were tested separately (Males: $F=26.15$, d.f. $=2, P<0.05$ and Females: $F=496.19$, d.f. $=2, P<0.05)$. There was no significant difference between the sexes, with a mean of $68 \%$ of males and $74 \%$ of females infected $(t=1.4$, d.f. $=17, P$ $>0.05)$.

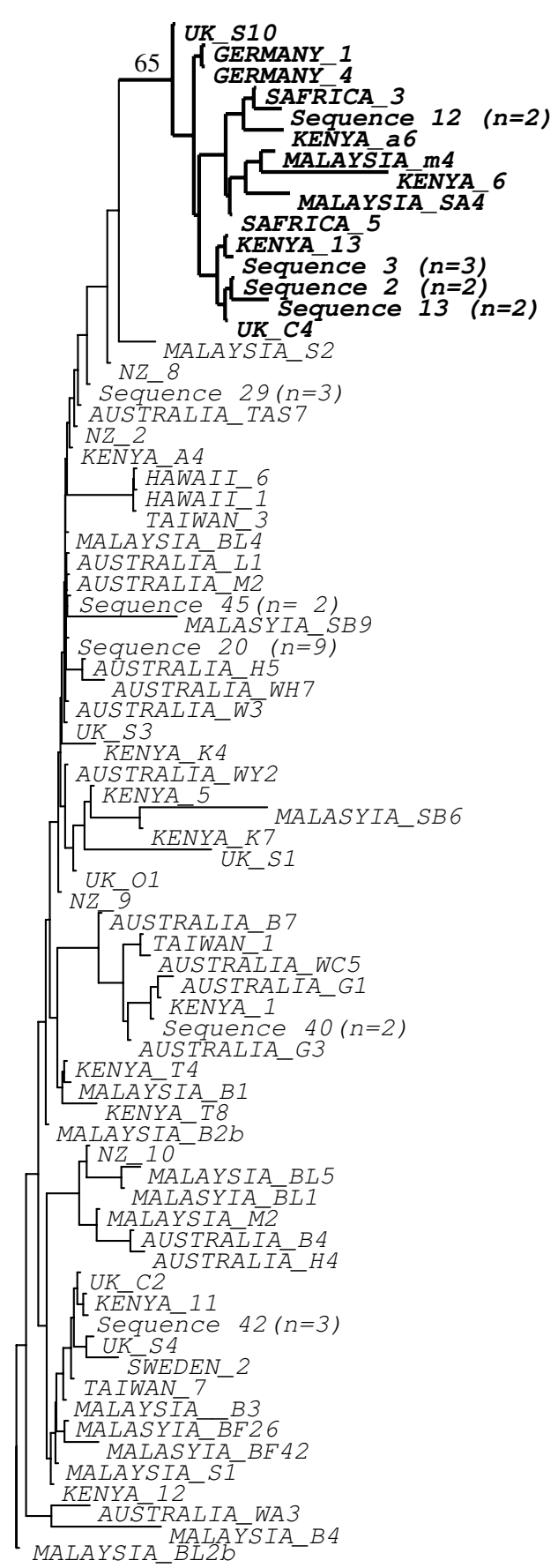

Figure 3

Phylogeny of DBM moths using nuclear data. Neighbour-joining tree of the 74 different Plutellaxylostella nuclear DNA (L27a gene) sequences. Numbers in brackets show multiple individuals with the same DNA sequence. The clade with bold labels and thick lines is supported by a major indel (see text) and has bootstrap support of 65. Country of origin is given for sequences found only in one moth. Sequences have been deposited in Genbank with accession numbers EU833259-833333. 
Table 3: Sex ratios and plutWBI infection frequencies in four Malaysian valleys.

\begin{tabular}{ccccc}
\hline Valley & $\mathbf{N}$ & Prop. Female & $\chi^{2}$ & Infected $(\mathbf{9 5} \% \mathbf{C I})$ \\
\hline Bertum & 145 & 0.63 & $4.79 *$ & $0.05(0.07)$ \\
Blue & 60 & 0.62 & 1.22 & $0.02(0.1)$ \\
Sungai Palas & 40 & 0.63 & 0.81 & $0(0.01)$ \\
Tringkapp & 48 & 0.60 & 0.67 & $0(0.1)$ \\
\hline
\end{tabular}

\section{Discussion}

\section{Wolbachia infections in P. xylostella}

We detected three different Wolbachia isolates in Plutella xylostella. The two A-clade isolates were very rare, each occurring in only two individual moths. The B-clade isolate plutWB1 was more common, but, where present in Malaysia and Kenya occurred at low frequencies of about $10 \%$. It is therefore likely that further screening in some of the other countries will also reveal plutWB1 (see Jiggins et al. 2001 for a discussion of sampling effort and Wolbachia detection). Interestingly, plutWB1 differs considerably in $w s p$ sequence from a previously reported B-clade infection ( $w X y l B 1)$ of P. xylostella in the USA [9]. It thus appears that P. xylostella has acquired different Wolbachia infections in different parts of its now global distribution. None of these infections appears common or widespread and some may be transient.

Interestingly, the whitefly Bemisia tabaci, another major crop pest with an essentially global distribution, has also been found to harbour a number of different Wolbachia infections [17]. The plutWB1 infection in P. xylostella is present in at least two continents and these insects probably stem from a single ancestral infection event, since their mtDNA haplotypes are very similar and form a well-supported and differentiated monophyletic group within the P. xylostella COI phylogeny (Figure 2). There is a small amount of variation in both the COI sequences of infected individuals (Figure 2) and the wsp sequences of their infections (Figure 1), reflecting a small amount of evolution since the original infection event.

\section{Phylogenies, infection and geography}

The host COI phylogeny has two major clades that correlate perfectly with presence or absence of the plutWB1 infection. The association between the very similar haplotypes 1-3 and plutWB1 infection supports the idea that
Wolbachia have driven this haplotype group to a significant if low frequency in infected populations. Our study further suggests that this infection is a sex ratio distorter and this phenotypic effect upon the host is likely to have driven the infection and associated haplotype group into the wider population. In contrast, there is little correlation between mtDNA haplotype and geographic origin. Infected and uninfected individuals from the same site in Malaysia (or Kenya) fall into the two different clades rather than group together.

In contrast, there is little tendency for individuals from the same country or even continent to cluster together in the mtDNA phylogeny (Figure 2). However, the lack of this pattern is consistent with recent and repeated movements of insects between continents due to trade in cruciferous crops. It is interesting that Australasia stands out as an exception to the general pattern. We sampled many Australian populations and two from New Zealand and all individuals belong to a single clade with very little mtDNA variation. This suggests that Australian and NZ populations probably stem from a single colonisation event from Asia around 120 years ago [18] that was then transferred from Australia to NZ or vice versa. The very low mtDNA diversity could reflect a small initial founder population from SE Asia. Low variation in the L27a gene (this study) and low microsatellite diversity [14] in Australian individuals further support this idea and suggest success of stringent quarantine procedures in modern times [14].

Finally, we note that selection for resistance to pesticides and biocontrol agents $[19,20]$ may also influence P. xylostella genetic variation. However, this would most likely influence both mtDNA and nuclear DNA, while our main result is a clear association of a sex-ratio distorting infection with patterns of mtDNA variation.

\section{Sex ratio distortion}

Our data show that the plutWB1 infection is associated with substantial sex ratio distortion, so that females outnumber males about two to one in infected lines. The fact that infection frequency was the same in males and females in our experiments argues against it being a male-killer. However, such a low infection frequency would be surprising for a feminizing bacterium, but is not unusual for a male-killer and host resistance to male-killing can evolve, as in the but-

Table 4: Sex ratios of infected laboratory lines over nine generations.

\begin{tabular}{ccccccc}
\hline Gen & $\begin{array}{c}\text { Line I7 } \\
\text { Females }\end{array}$ & Males & Prop. Fem. & $\begin{array}{c}\text { Line 95 } \\
\text { Females }\end{array}$ & Males & Prop. Fem. \\
\hline I to 3 & 73 & 23 & 0.76 & 253 & 88 & 0.74 \\
4 to 6 & 545 & 218 & 0.71 & 408 & 242 & 0.63 \\
7 to 9 & 209 & 142 & 0.60 & 224 & 121 & 0.65 \\
\hline
\end{tabular}


terfly Hypolimnas bolina [21]. Male-killing alone cannot explain the sex ratio bias in our infected lab lines and feminisation of genetic males is an interesting possibility for future study. It is also possible that a more complicated scenario could apply and that Wolbachia is not, or not the only, agent of sex ratio bias [22]. Our evidence for the role of Wolbachia is currently only correlational, which is an important but not definitive line of evidence [22]. Recent work has revealed that other less common, but still widespread [23] insect endosymbionts can cause similar hosts effects to Wolbachia [23,24]. Furthermore, detailed analysis of a case involving Ostrinia moths, initially thought to involve a feminising Wolbachia isolate, has revealed complex fitness interactions between sex chromosomes and infection status leading to differential mortality by sex [25].

\section{Conclusion}

Global patterns of mtDNA variation in the diamondback moth are shaped largely by the presence or absence of the plutWB1 Wolbachia infection. This occurs patchily at low frequencies and apparently distorts host sex ratios to a 2:1 bias in favour of females. The cause and mode of sex ratio distortion deserves further study. In contrast to infection status, the geographic origin of a given individual is a poor predictor of its mtDNA haplotype, due to recent human transport of insects between continents in vegetable crops.

\section{Methods}

\section{Field sampling of insects on four continents}

Between 2001-2006 we sampled Plutella xylostella individuals from 33 sites in 10 countries: Australia, New Zealand, Malaysia (Peninsular and Sabah), Kenya, South Africa, United Kingdom, Taiwan, Sweden, Germany and Hawaii. We also included three Plutella porrectella individuals from the UK as outgroups. Insects used were either final instar larvae or adults and were field caught individuals or their F1 offspring (except Sweden, South Africa and UK Oxford, which were laboratory cultures). Insects were stored in $70-100 \%$ ethanol for preservation of DNA.

\section{DNA extraction}

To minimise the risk of contamination, the pre-extraction treatment and all DNA extractions were performed under sterile conditions After taking insects out of their collection tubes, each individual was cleaned by immersion in $70 \%$ ethanol, followed by two rinses in double-distilled DNA/ RNA free water and then allowed to dry for $5 \mathrm{~min}$. Larvae were dissected and checked for endoparasites. DNA was then extracted by grinding the abdomen of adults, or the entire larva, in 200 ul of 5\% Biorad Chelex 100 resin solution in the presence of proteinease $\mathrm{K}(12 \mu \mathrm{g} / \mu \mathrm{l})$, followed by $3 \mathrm{~h}$ incubation at $55^{\circ} \mathrm{C}$ and $15 \mathrm{~min}$ boiling at $96^{\circ} \mathrm{C}$. The samples were then centrifuged and stored at $-20^{\circ} \mathrm{C}$ until use. For each set of extractions a blank extraction was performed using all the reagents minus the DNA extract (this was performed at least once for every 10 insects).

\section{Wolbachia screening and sequencing}

We tested 306 individuals for the presence of Wolbachia infection with PCR using extended versions of the Wolbachia specific primers ftsZf1 5' GTT GTC GCA WTA CYG ATG CTC A 3' and ftsZr1 5' CTT AAG TAA GCT GGT ATA TCA ATA 3' [26] to amplify an approximately $1000 \mathrm{bp}$ stretch of the Fts $Z$ gene encoding the bacterial cell cycling gene. We scored individuals yielding a product of the expected size as provisionally infected, and samples that did not amplify as provisionally uninfected.

DNA extracts that tested positive for $f t s Z$ were then double-checked by performing a PCR using a different set of Wolbachia specific primers (wsp81F and wsp691R) [27], which amplify the wsp (Wolbachia Surface Protein) gene and yield a product of approximately $550 \mathrm{bp}$. All samples that were positive for $f t s Z$ also tested positive using the $w s p$ primers. We later sequenced these $w s p$ products.

Provisional negative samples were tested for the quality of DNA extract by performing PCR for part of the insect mitochondrial cytochrome oxidase I (CO1) gene. We used C1-J-2183 (alias Jerry) and L2-N-3014 (alias Pat) primers, which yield an approximately 1000 bp product [28]. If CO1 PCR was successful, we regarded the sample as uninfected. If it failed, we excluded the sample from our data. DNA extracts that scored as Wolbachia infected were also amplified for the CO1 gene. All the CO1 genes amplified were later sequenced.

Finally, a segment of the nuclear L27a gene, encoding the ribosomal protein L27a was amplified using the primers L27aFor1 5' ACG GTC ATG GAC GTA TCG GTA A 3' and L27aRev2 5' ATG TTG ATG ACT GGC ACC TTG C 3' (S. Baxter, pers. comm.) to yield an approximately $200 \mathrm{bp}$ product, which was later sequenced.

The PCR temperature profile for fts $Z$ was $95^{\circ} \mathrm{C}$ for $30 \mathrm{sec}$, $55^{\circ} \mathrm{C}$ for $1 \mathrm{~min}$ and $72^{\circ} \mathrm{C}$ for $1 \mathrm{~min}$, for a total of 35 cycles, and final elongation time of $7 \mathrm{~min}$ at $72^{\circ} \mathrm{C}$. We used the same conditions for $w s p$, L27a and CO1 (annealing temperature reduced to $50^{\circ} \mathrm{C}$ ). We electrophoresed 25 ul of each PCR product on a $1 \%$ agarose gel to determine amplicon presence and size. We excised gel bands and purified them using a GFX DNA Purification Kit (Amersham Pharmacia Biotech Inc.), before sequencing directly in both directions using the PCR primers. Sequences were obtained using the ABI PRISM Big Dye Terminator Cycle Sequencing Kit (Perkin Elmer Inc.) and the ABI Prism 3700 DNA Analyser (Perkin Elmer Inc.) and assembled using Sequencher ${ }^{\mathrm{TM}}$ (Gene Codes Cooperation).

\section{Phylogenetic analysis}

Sequences were aligned manually using SE-AL v2.0a11 Carbon [following previous alignments and excluding the third hypervariable region in the case of $w s p[9,27,29]]$. 
There were no gaps in the CO1 sequence alignment of $P$. xylostella samples. There was a 9 bp gap that started at base 182 in the L27a sequence alignment. All phylogenies were estimated using PAUP* version 4.0b [30].

To determine the phylogenetic affinities of the Wolbachia isolates we constructed a neighbour-joining tree [31] of the $w s p$ sequences. We adopted the standard criterion of 2.5\% wsp sequence divergence to distinguish between Wolbachia isolates [27]. Isolates differing by this amount should be discrete infections, although recent work shows that infections with similar sequences in one gene may differ considerably in other genes, making a multi-locus strain typing (MLST) approach necessary to show close identity of apparently similar isolates from different hosts [32,33].

MtDNA (CO1) phylogeny was reconstructed using NJ and MP methods. MP trees were reconstructed using the method of Quicke et al. [34] by conducting an initial heuristic search of 10,000 random additions, tree-bisectionreconnection (TBR) branch swapping, and holding one tree per replicate. We then used the trees generated by the initial search as starting trees for a second heuristic search, in which we saved multiple trees. We assessed clade support using 1000 bootstrap replications.

We used MODELTEST 3.06 [35] to select nucleotide substitution models for $\mathrm{NJ}$ trees. The model $\mathrm{HKY}+\mathrm{G}$ was selected for $w s p$ and $\mathrm{CO} 1$. Bootstrap values were generated from 10,000 replicates. We rooted the mtDNA phylogeny using the outgroup (Plutella porrectalla).

\section{MtDNA haplotype analysis}

Haplotype number, haplotype diversity and nucleotide diversity $(\pi)$ [36-38] were calculated for five geographic regions: 1. Australasia (Australia/New Zealand), 2. Africa (Kenya/South Africa), 3. Europe (UK, Germany, Sweden), 4. Asia (Malaysia, Taiwan) and 5. Hawaii, and also for matched infected and uninfected groups (Tables 2 \&3). Haplotype diversity describes the number and frequency of different haplotypes and nucleotide diversity is defined as the average number of pair-wise nucleotide difference per site. Haplotype and nucleotide diversity measurements are appropriate for this type of data because they do not depend on the length of DNA fragment or sample size [36], unlike the number of pairwise nucleotide differences or haplotypes.

\section{Phenotypic effects of plutWB I}

We collected individuals from four valleys in the Cameron Highlands, Malaysia in May 2004 to obtain data on field sex ratios and insects to establish isofemale laboratory lines. We scored 293 individuals collected as last instar larvae or pupae and then reared to adulthood. The valleys are separated by forested mountain ridges and consist of farms that grow commercial Brassica plants and other vegetables.

We established two plutWB1 infected lines (17 and 95) and three uninfected ones $(26,61$ and 86) from the fieldsampled insects by mating a virgin male with a virgin female individual. Each line was kept in a rectangular, transparent container $(116 \times 8 \times 7 \mathrm{~cm} \mathrm{~L} \times \mathrm{W} \times \mathrm{D})$ and containing a cotton-wool ball soaked in $10 \%$ honey solution and an egg-laying sheet (tin foil, $13 \times 5 \mathrm{~cm}$ ) dipped in cabbage solution ( $65 \mathrm{~g}$ cabbage per $500 \mathrm{ml} \mathrm{ddH_{2 }}$ ) for 3 days. We then reared the eggs produced by each pair to the pupal stage on Brassica napus seedling trays $(50 \times 25 \times 6$ $\mathrm{cm}$ ) in cages with a metal cube frame, covered in fine netting. To avoid overcrowding, no more than 100 larvae were reared per seedling tray.

To continue each isofemale line, pupae were collected and placed in individual tubes and checked daily for emergence of adults. At least 100 individuals (50 males and 50 females) from each isofemale line were then placed in containers (with an egg laying sheet and honey solution) for 3 days. Subsequently, the eggs were reared and the isofemale line continued as above.

In each generation the sex ratio of each line was recorded by counting the total number of male and females. The prevalence of plutWB1 was estimated by screening at least 10 males and 10 females per line. However, low numbers reduced sample size in two lines $(17,95)$ in generations one and two. The lines were kept in a controlled environment room, with a 16:8 light: dark cycle, at $23 \mathrm{C}$ and 50$75 \%$ humidity.

\section{Statistical analysis of the phenotypic effects of Wolbachia} We first tested whether plutWB1 infection was associated with host sex ratio distortion and then whether there was evidence for male-killing. Since both sex ratio and infection status are binomial variables yielding proportion data, we arcsin transformed $[\arcsin (\sqrt{\text { proportion }}(p)]$ these variables. Data analysis was performed in Excel with the Analysis-It software.

We tested for departures from a 1:1 sex ratio in field populations using the Chi-squared test on data from individual and pooled valleys in the Cameron Highlands, Malaysia. The same test for deviation from 1:1 was also used for sex ratios of infected and uninfected isofemale lines.

We tested for a significant difference in sex ratio between infected and uninfected lab lines. We first used an unpaired t-test to examine if there was any significant difference in the sex ratio between infected and uninfected 
lines, using the total numbers of males and females in all nine generations for each line. We then examined whether infected lines varied across generations, by comparing the sex ratios of early ( 1 to 3 ), mid (4-6) and late (7 to 9) generations using a repeated measures ANOVA. Finally, we confirmed that there was no difference between the two infected lines using a paired t-test $(t=0.46$, d.f. $=8, P>$ $0.05)$.

For infection frequency, we first confirmed that there was no difference between the two infected lines, using a paired t-test $(t=1.51$, d.f. $=8, P>0.05)$. This then allowed us to use the two lines as independent replicates in a repeated measures ANOVA to test if infection frequency changed between early, mid and late generations (see above). We conducted this analysis for males and females separately. Finally, we used a t-test on all males and all females to test for a sex difference in infection frequency. This focus on differences between the sexes is because a male-killing Wolbachia infection is expected to occur at (much) lower frequency in males than in females, unless effective host resistance has evolved.

\section{Abbreviations}

(MP): Maximum parsimony; (NJ): neighbour-joining; (TBR): tree-bisection-reconnection.

\section{Authors' contributions}

JMC conceived and $\mathrm{AD}$ designed the project. $\mathrm{AD}$ carried out the field and laboratory practical work and analysed the data. JMC and $\mathrm{AD}$ wrote the paper and both authors read and approved the final manuscript.

\section{Acknowledgements}

We thank C. Godfray, M. Bonsall, R. Kfir, R. Gothu, D. Leoni, M. Chou, M. Hommes, G. Walker, B. Ekbom, N. Talekar, D. Slade, and B. Lohr for contributing moth samples, and especially Nancy Endersby for sending us large samples of insects from Australia. We also thank S. Baxter for sharing unpublished primer data and Ellie Haine and two anonymous referees for comments on the manuscript. This work was supported financially by the BBSRC and NERC.

\section{References}

I. Avise J: Phylogeography: the history and formation of species. Harvard University Press, Cambridge, Massachusetts; 2000.

2. Hurst G, Jiggins FM: Problems with mitochondrial DNA as a marker in population, phylogeographic and phylogenetic studies: the effects of inherited symbionts. Proceedings of the Royal Society London B 2005, 272: I525-1534.

3. Werren $\mathrm{JH}$, Windsor DM: Wolbachia infection frequencies in insects: evidence of a global equilibrium. Proceedings of the Royal Society London B 2000, 267: I277-I 285.

4. Hilgenboecker K, Hammerstein P, Schlattmann P, Telschow A, Werren JH: How many species are infected with Wolbachia? - a statistical analysis of current data. FEMS Microbiology Letters 2008, 28I(2):215-220.

5. Bourtzis K, Miller T: Insect Symbiosis. Boca Raton [FL]: CRC Press; 2003.

6. O'Neill S, Hoffmann A, Werren J: Influential Passengers: Inherited Microorganisms and Arthropod Reproduction. New York: Oxford University Press; 1997.
7. Sasaki T, Massaki N, Kubo T: Wolbachia variant that induces two distinct reproductive phenotypes in different hosts. Heredity 2005, 95(5):389-393.

8. Shoemaker DD, Keller G, Ross KG: Effects of Wolbachia on mtDNA variation in two fire ant species. Molecular Ecology 2003, I 2: 1757-I77|.

9. Jeyaprakash A, Hoy MA: Long PCR improves Wolbachia DNA amplification: wsp sequences found in $76 \%$ of sixty-three arthropod species. Insect Molecular Biology 2000, 9(4):393-405.

10. Talekar NS, Shelton AM: Biology, ecology, and management of the diamondback moth. Annual Review of Entomology 1993, 38:275-301.

II. Harcourt D: Biology of cabbage caterpillars in eastern Ontario. Proceedings of the Entomological Society of Ontario 1963, 93:6I-75.

12. Kfir R: Origin of the diamondback moth (Lepidoptera: Plutellidae). Annals of the Entomological Society of America 1998, 91:164-167.

13. Chapman J, Reynolds D, Smith A: High altitude migration of the diamondback moth Plutella xylostella to the UK: a study using radar, aerial netting and ground trapping. Ecological Entomology 2000, 27:64I-650.

14. Endersby NM, McKechnie SW, Ridland PM, Weeks AR: Microsatellites reveal a lack of structure in Australian populations of the diamondback moth, Plutella xylostella (L.). Molecular Ecology 2006, I5: 107-II8.

15. Haine ER, Cook JM: Convergent incidences of Wolbachia infection in fig wasp communities from two continents. Proceedings of the Royal Society London B 2005, 272:42I-429.

16. Jiggins FM, Hurst GDD, Dolman CE, Majerus MEN: High-prevalence male-killing Wolbachia in the butterfly Acraea encedana. Journal of Evolutionary Biology 2000, I3:485-50I.

17. Nirgianaki A, Banks GK, Frohlich DR, Veneti Z, Braig HR, Miller TA, Bedford ID, Markham PG, Savakis C, Bourtzis K: Wolbachia infections of the whitefly Bemisia tabaci. Current Microbiology 2003, 47(2):93-10I.

18. Tyron H: Report on Insect and Fungus Pest No. I. Department of Agriculture, Queensland, James C. Beal. Government Printer, Brisbane, Australia; 1889.

19. Heckle D, Gahan LJ, Tabashnik BE, Johnson M: Randomly amplified polymorphic DNA differences between strains of diamondback moth (Lepidoptera: Plutellidae) susceptible or resistant to Bacillus thuringiensis. Annals of the Entomological Society of America 1995, 88:531-537.

20. Tabashnik BE, Cushing N, Finson N, Johnson M: Field development of resistance to Bascillus thuringiensis in diamondback moth (Lepidoptera: Plutellidae) resistance to insecticides in Hawaii: intra-island variation and cross-resistance. Journal of Economic Entomology 1990, 80:1091-1099.

21. Hornett EA, Charlat S, Duplouy AMR, Davies N, Roderick GK, Wedell N, Hurst GDD: Evolution of Male-Killer Suppression in a Natural Population. Plos Biology 2006, 4(9):e283. doi: I0.137 I/ journal.pbio.0040283

22. Weeks AR, Reynolds KT, Hoffmann AA, Mann H: Wolbachia dynamics and host effects: what has (and has not) been demonstrated? Trends in Ecology \& Evolution 2002, 17(6):257-262.

23. Weeks AR, Velten R, Stouthamer R: Incidence of a new sex-ratiodistorting endosymbiotic bacterium among arthropods. Proceedings of the Royal Society of London Series B-Biological Sciences 2003, 270(I526): 1857-1865.

24. Weeks AR, Stouthamer R: Increased fecundity associated with infection by a Cytophaga-like intracellular bacterium in the predatory mite, Metaseiulus occidentalis. Proceedings of the Royal Society of London Series B-Biological Sciences 2004, 27 I:S193-SI 95.

25. Kageyama D, Traut W: Opposite sex-specific effects of Wolbachia and interference with the sex determination of its host Ostrinia scapulalis. Proceedings of the Royal Society of London Series B-Biological Sciences 2004, 27 I (I 536):25I-258.

26. Werren JH, Zhang W, Guo LR: Evolution and Phylogeny of Wolbachia: reproductive parasites of arthropods. Proceedings of the Royal Society London B 1995, 26 I:55-7I.

27. Zhou W, Rousset F, O'Neill S: Phylogeny and PCR-based classification of Wolbachia strains using wsp gene sequences. Proceedings of the Royal Society London B 1998, 265:509-5I5.

28. Simon C, Frati F, Beckenbach A, Crespi B, Liu H, Flook P: Evolution, Weighting, and Phylogenetic Utility of Mitochondrial Gene 
Sequence and a compilation of Conserved Polymerase Chain Reaction Primers. Annals of the Entomological Society of America 1994, 87(6):65I-70I.

29. Shoemaker DD, Machado CA, Molbo D, Werren JH, Windsor DM, Herre EA: The distribution of Wolbachia in fig wasps: correlations with host phylogeny, ecology and population structure. Proceedings of the Royal Society London B 2002, 269:2257-2267.

30. Swofford D: PAUP* Phylogenetic Analysis Using Parsimony (*and Other Methods). Sunderland [MA]: Sinauer Associates; 2002.

31. Saitou N, Nei M: The neighbour-joining method: a new method for reconstructing phylogenetic trees. Molecular Biology and Evolution 1987, 4: I406-1425.

32. Baldo L, Dunning Hotopp JC, Jolley KA, Bordenstein SR, Biber SA, Choudhury RR, Hayashi C, Maiden MCJ, Tettelin H, Werren JH: Multilocus Sequence Typing System for the Endosymbiont Wolbachia pipientis. Appl Environ Microbiol 2006, 72(I I):7098-7I I0.

33. Baldo L, Werren JH: Revisiting Wolbachia supergroup typing based on WSP: Spurious lineages and discordance with MLST. Current Microbiology 2007, 55(I):8I-87.

34. Quicke D, Taylor J, Purvis A: Changing the landscape: a new strategy for estimating large phylogenies. Systematic Biology 200I, 50:60-66.

35. Posada D, Crandall K: MODELTEST: testing the model of DNA substitution. Bioinformatics 1998, I4:817-818

36. Nei M: Molecular Evolutionary Genetics. New York: Columbia University Press; 1987.

37. Nei M, Li W: Mathematical model for studying genetic variation in terms of restriction endonuclease. Proceedings of the National Academy of Sciences USA 1979, 76:5269-5273.

38. Tajima F: Evolutionary relationship of DNA sequences in finite populations. Genetics 1983, 105:437-460.
Publish with Bio Med Central and every scientist can read your work free of charge

"BioMed Central will be the most significant development for disseminating the results of biomedical research in our lifetime. "

Sir Paul Nurse, Cancer Research UK

Your research papers will be:

- available free of charge to the entire biomedical community

- peer reviewed and published immediately upon acceptance

- cited in PubMed and archived on PubMed Central

- yours - you keep the copyright 